\title{
Maduromycosis of Hand: A Rare Form of Chronic Granulomatous Infection with Role of Imaging
}

Sanjay Nathani ${ }^{1,2^{*}}$ and Pooja Mehta ${ }^{2}$

${ }^{1}$ Department of Radiodiagnosis Medipulse Hospital Jodhpur, Rajasthan, India

${ }^{2}$ Department of Radiodiagnosis, SRL, SS Wellness and Research Center Jodhpur, Rajasthan, India

*Corresponding author: Sanjay Nathani, Department of Radiodiagnosis, Medipulse Hospital and SRL, 2S Wellness and Research Center, Jodhpur, Rajasthan, India, 342005, Tel: (+91)9783217075, (+91)9462772596; E-mail: drsanjaynathani2006@gmail.com

Received date: July 21, 2018; Accepted date: August 08, 2018; Published date: August 20, 2018

Copyright: (C) 2018 Nathani S, et al. This is an open-access article distributed under the terms of the Creative Commons Attribution License; which permits unrestricted use; distribution; and reproduction in any medium; provided the original author and source are credited.

\begin{abstract}
Maduromycosis disease, also called as mycetoma, is a form of chronic granulomatous infection caused by either actinomycetes (Actinomycotic mycetoma) or true fungi (Eumycotic mycetoma) following a trivial penetrating injury. According to the literature and published reports, so far maduromycosis of the hand is less common than involvement of foot (Madura foot). The disease is slowly progressive in nature, often presenting at later stage with complications and deformities due to painless course, lack of patient awareness at earlier stages of disease, poor response to routine drug therapy and limited experience of treating physician about this rare entity. We are presenting here a case of middle-aged farmer from lower socio-economic strata who presented with deformed right hand, small ulcerations, and induration over scarred discolored skin and discharging sinuses after thorn injury followed by spreading infection of the soft tissue and bones described as maduramycosis disease.
\end{abstract}

Keywords: Mycetoma; Maduromycosis; Hand

\section{Introduction}

Historically, Gill first described the disease 'mycetoma of foot' in 1842 and named it "Madura Foot" after the place of its detection in Madura district of South India region [1]. Foot is the most common site of infection due to its susceptibility to trauma but the disease can occur in any other regions of the body including hand. It is a chronic granulomatous type of infection that develops after a penetrating injury causing inoculation of the causative agent that eventually spreads the disease into the surrounding soft tissues and osseous structures [2]. Clinically the disease is characterized by localized swelling/tumefaction, nodules formation, multiple draining sinuses and expulsion of pigmented granules in discharge with overlying skin indurations/discoloration [3]. We are presenting here a case report of advanced maduromycosis of hand with classical clinical and imaging findings.

\section{Case Description}

A 35-year-old male farmer presented with deformity and swelling of right-hand, discoloration of skin and multiple discharging sinuses without definite history of pigmented granule expulsion. On recollection, there was history of a penetrating injury by a thorn few months back. Frontal and oblique radiographs of right-hand showed cortical irregularity, infiltrating destruction and irregular periosteal reaction involving second and third metacarpals and proximal phalanx of second finger with large soft tissue swelling around them. Contrastenhanced magnetic resonance imaging (MRI) examination of righthand using flexible 4-channel coil on 1.5 Tesla machine showed T2W heterogeneously hyperintense soft tissue in interosseous planes encasing second and third metacarpals with cortical irregularity and few well marginated $\mathrm{T} 2$ hypointense rim lesions at places showing mild post-contrast enhancement. On the basis of clinical and imaging findings correlating with penetrating injury, diagnosis of mycetoma infection was made with possible diagnostic differential of hemangioma due to atypical imaging appearance of the disease in this particular case. On surgery and histopathology, diagnosis of mycetoma was confirmed (Figures 1-3).

\section{Images}

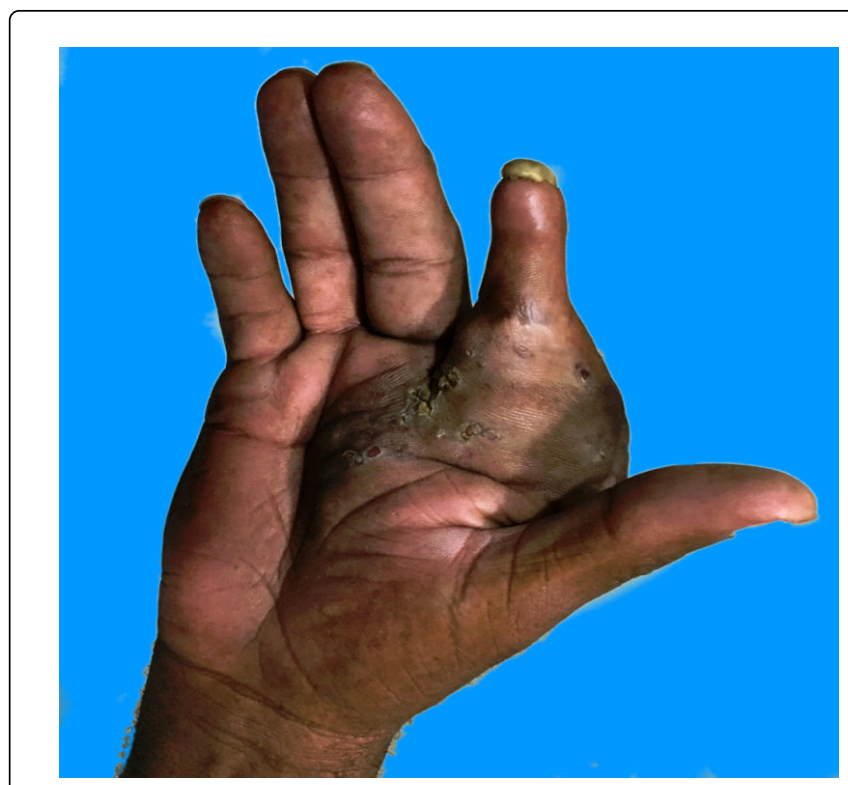

Figure 1: Clinical image of right-hand with deformity, swelling, discoloration of skin and multiple discharging sinuses. 


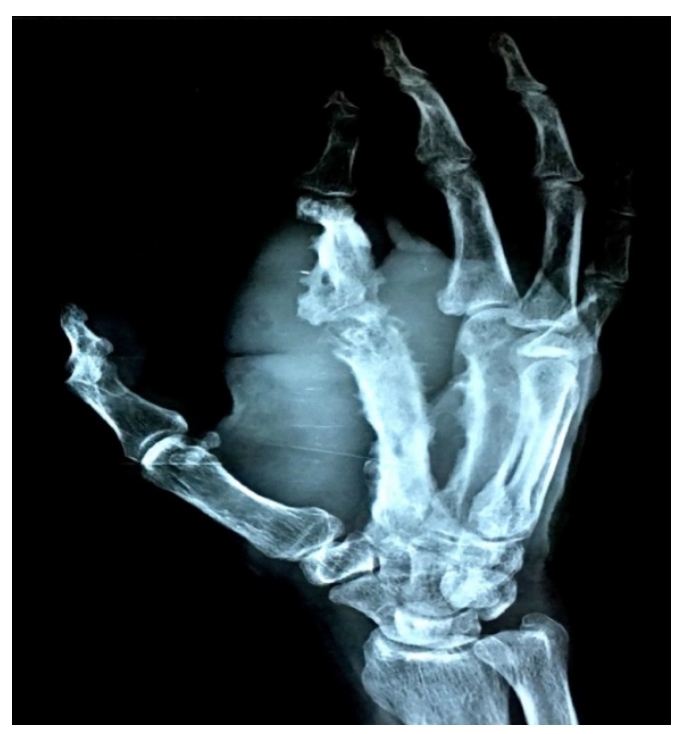

Figure 2: Frontal and oblique radiographs of right hand showing cortical irregularity, destruction and irregular periosteal reaction involving second and third metacarpals and proximal phalanx of second finger with associated large soft tissue swelling, also involving web space.
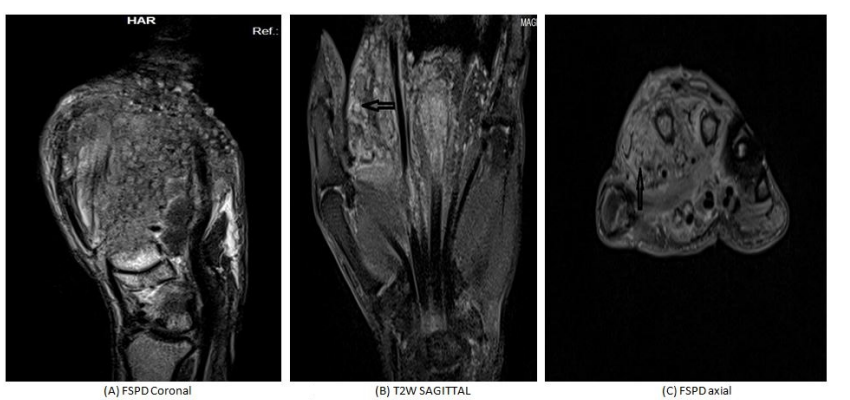

Figures 3: Magnetic resonance imaging of right hand (A) Fatsuppressed proton density (FSPD) coronal, (B) T2W Sagittal and (C) FSPD axial images showing diffuse infiltration of first to $3 \mathrm{rd}$ web space and interosseous muscles by large altered signal intensity soft tissue lesion causing erosive changes and cortical irregularity of underlying bones of 2 nd and 3rd fingers associated with multiple abscesses, spherical hyperintense lesions having internal hypointense foci ("dot-incircle"sign-arrow mark) and discharging sinuses.

\section{Discussion}

Maduromycosis is a slowly progressive infective chronic granulomatous disease affecting the skin and subcutaneous tissues with potential of bony involvement, destruction, deformity and formation of discharging sinuses by the causative organism, true fungi (Eumycetoma) or the bacteria (Actinomycetoma) [4]. The disease is endemic in tropical and subtropical regions, predominantly, India and Africa, with prevalence of Actinomycetoma in areas of abundant rainfall as compared to Eumycetoma, which is more prevalent in areas with scarce rainfall $[5,6]$. The clinical presentations are similar and final diagnosis can been achieved by genomic technique. The disease is more common amongst the male of lower socio-economic strata during $2^{\text {nd }}$ to $5^{\text {th }}$ decades, especially farmers with a tendency to work barefoot [7]. The disease process is initiated by introduction of the causative organism via localized trauma of the skin, usually trivial like with thorns, or implantation with solid objects harbouring organisms on the surface [8]. The most common locations being foot and leg, however, hand or any other anatomical site may also be affected. At the earlier stage patients present with small non-tender skin nodules, ulcerations and/or discharging sinus tracts. Exudate containing granules may be seen from discharging sinuses that may be of yellow, white, red, brown or black, depending on the causative agent. The skin overlying the affected area generally becomes indurated and discoloured. If left untreated, the disease process is complicated by the involvement of adjacent bones with tumefaction, soft tissue abscess formation and deformity of the limb [9]. Eumycetomas are usually more localized than Actinomycetomas. Differential diagnoses of maduromycosis are mainly chronic granulomatous diseases including tuberculosis, leprosy and syphilis; however, uncommon conditions like Botryomycosis, tropical ulcer and locally aggressive soft tissue neoplasm are also kept in this category before making the final diagnosis.

Role of various imaging modalities has been described in the diagnosis of maduromycosis including radiographs, ultrasonography, computed tomography (CT) and MRI to make proper diagnosis, evaluate the extent of the disease and serve as a roadmap to surgery. At an earlier stage, radiographs of infected area may appear normal. With the progression of disease various imaging findings such as soft tissue swelling, lytic erosive changes in infected bone and irregular periosteal reaction may be seen as observed in our case. Scalloping of bone and reduced or increased bone density has also been described. Maduromycosis classically first involves the outer or cortical aspect of bone as opposed to bacterial osteomyelitis that tends to begin from medullary cavity [10]. Eumycotic disease form large bone cavities of $\geq 1$ $\mathrm{cm}$ in diameter and Actinomycetes form multiple sub-centimeter-sized cavities having moth-eaten appearance [9]. CT is better for depiction of bony changes than radiographs.

Role of "dot-in-circle" sign on USG and MRI has been described by Sarris et al. [11] as a highly specific finding in case of mycetoma. On ultrasonography, "dot-in-circle" sign is seen as thick-walled round or oval hypoechoic lesions with internal tiny echogenic foci; increased vascularity at periphery may be demonstrated due to inflammatory changes on color Doppler. Similarly, MRI shows multiple abscesses and spherical to oval T2-weighted iso-to-hyperintense signal lesions with internal hypointense foci surrounded by inflammatory or granulation tissue showing relatively hypointense signal; post-contrast study shows enhancement of the lesions with persistent central hypointense foci. With progression cortical irregularity and marrow signal abnormality are seen in adjacent bones with infiltration of soft tissue and presence of discharging sinuses. MRI imaging is considered superior and helps in early diagnosis, to outline the extent of the disease process and do preoperative planning. Histopathological evaluation of discharging sinuses and pigmented granules is helpful in confirming the diagnosis by evidence of fungal hyphae or bacterial colonies and granulomatous component. Various microbiological staining agents are used to differentiate actinomycetoma and eumycetoma, e.g. Gram stain, Gomori methenamine silver, periodic acid-Schiff and lactophenol blue $[12,13]$. 
Page 3 of 3

People living in endemic areas, especially in developing countries should be educated by the appropriate local health care professionals or medical service providers about the precautionary measures to avoid activities and risk factors that expose them to infective agents like barefoot walking, avoid handling of soiled wooden sticks with thorny branches or cow dung handling with bare hands, use of protection like gloves, shoes or sandals while working in fields and washing of limbs at regular intervals to protect themselves.

Treatment of maduromycosis depends on the severity and extent of the disease evaluated radiologically and clinically with confirmation of the definitive causative agent by histopathology. Maduromycosis responds to various combinations of antibiotics, antifungal and antimicrobial agents and surgery is needed in patients with locally respectable advanced disease with deformity and extensive bone involvement not responding to medical management. The management of maduromycosis is difficult and unsatisfactory due to its long duration to achieve cure, potential side-effects of drugs, high recurrence rate and in terms of cost burden to the patients and health authorities.

Role of health care providers is utmost important in endemic areas to prevent the infections, educate the people usually belonging to lower socio-economic scales, especially in developing countries, motivate patients about regular drug intake, interval follow up and assessment for disease progression. This can be constructive in preventing deformities and need of amputations. A constant interaction between local health care professionals or medical service providers and patients is required in such chronic diseases due to their long course of treatment and high relapse rates.

\section{Conclusion}

Aim of this article is to achieve early diagnosis of maduromycosis with the help of its clinical and radiological appearance to make the physician and radiology fraternity aware about the disease signs in endemic areas, often misdiagnosed in the early stage as soft tissue tumors as well as chronic bacterial or tuberculosis infections, when typical discharging sinuses are not noted. With the help of certain clinical and radiological features, this disease can be easily diagnosed at an early stage and achieve prevention of residual limb deformity or surgeries/amputations.

\section{References}

1. Gill (1874) Indian army medical reports. Churchill, London.

2. Rai V, Balacharan C (2006) Actinomycotic mycetoma of hand: A rare occurrence. Indian J Dermatol Venereol Leprol 72: 178.

3. Foltz KD, Fallat LM (2004) Madura foot: Atypical finding and case presentation. J Foot Ankle Surg 43: 327-31.

4. Negroni R, Lopez DG, Arechavala A, Bianchi MH, Robles AM (2006) Clinical and microbiological study of mycetomas at the Muniz Hospital of Buenos Aires between 1989 and 2004. Rev Argent Microbiol 38: 13-18.

5. Welsh O (1993) Mycetoma. Semin Dermatol 12: 290-295.

6. Bakshi R, Mathur DR (2008) Incidence and changing pattern of mycetoma in western Rajasthan. Indian J Pathol Microbiol 51: 154-155.

7. Magana M (1984) Mycetoma. Int J Dermatol 23: 221-236.

8. McGinnis MR, Fader RC (1988) Mycetoma: A contemporary concept. Infect Dis Clin North Am 2: 939-954.

9. Lewall DB, Ofole S, Bendl B (1985) Mycetoma. Skeletal Radiol 14: 257-262.

10. Abd El, Bagi ME (2003) New radiographic classification of bone involvement in pedal mycetoma. AJR Am J Roentgenol 180: 665-668.

11. Sarris I, Berendt AR, Athanasous N, Ostlere SJ (2003) MRI of mycetoma of the foot: Two cases demonstrating the dot-in-circle sign. Skeletal Radiol 32: 179-183.

12. Magana M (1994) Mycetoma, some clinical and histopathological features. Turk J Dermatopathol 3: 94.

13. Pilsczek FH, Augenbraun M (2007) Mycetoma fungal infection: Multiple organisms as colonizers or pathogens. Rev Soc Bras Med Trop 40: 463-465. 